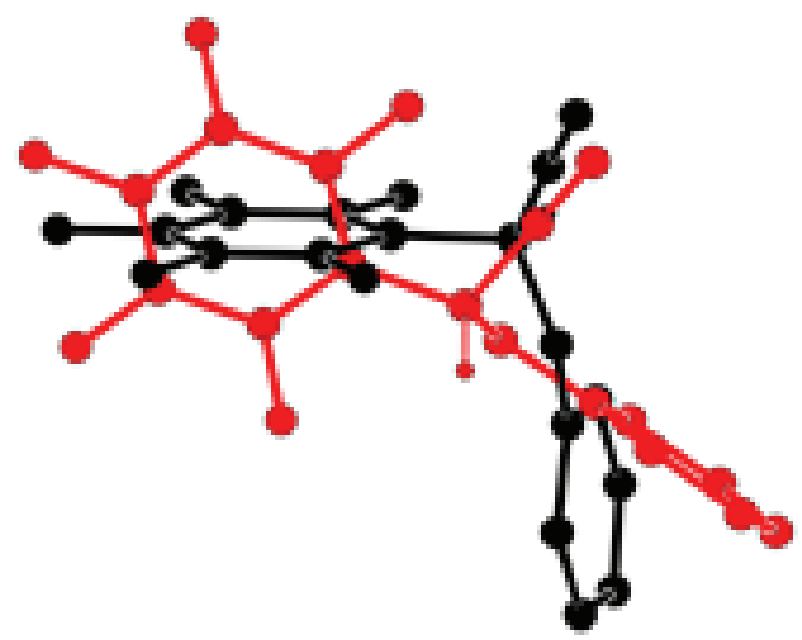

Figure 1.

Keywords: kryptoracemate, false conglomerate, chiral solids, polymorphysm, intermolecular interactions, halogen bonding, $\mathrm{H}$ bonding, $\pi$ stacking interactions

\section{MS36-P15 A technique for the comparison and analysis of decorated molecular surfaces}

Peter R. Spackman ${ }^{1}$, Dylan Jayatilaka ${ }^{1}$

1. University of Western Australia

email:20265845@student.uwa.edu.au

Molecular surfaces of various types are of immense value in chemistry. Typically, comparison and analysis of such surfaces relies upon human intuition and understanding which is inherently subjective.

We present a technique to describe the shape of the surface, and any associated properties on the surface (so-called decorations) which preserves relative location information while remaining translation and rotation invariant. The technique relies on a spherical harmonic decomposition followed by manipulation of the expansion coefficients in order to generate any number of rotation-invariant descriptors. [1] Although others have employed spherical harmonic decompositions for shape (see [2], [3]), the generation of rotation-invariant descriptors for shape and properties mapped on the surface is new in this context.

Importantly, the technique provides the capability to greatly reduce the amount of information which is invaluable for efficient large-scale algorithmic classification. An example of shape reconstruction is shown Figure 1 dependent only on the highest angular momentum $l_{\max }$ of the spherical harmonics used in the process.

The technique will be applied on several sets of decorated Hirshfeld surfaces. Conclusions will be drawn from cluster analysis to objectively examine questions related to, for example, shape and interaction-dependent factors affecting crystal packing; and binding-pocket correlations for drug-search and co-crystallisation applications.

\section{References}

1. Burel, G. and H. Henocq, 3-DIMENSIONAL INVARIANTS AND THEIR APPLICATION TO OBJECT RECOGNITION. Signal Processing, 1995. 45(1): p. 1-22.

2. Morris, R.J., et al., Real spherical harmonic expansion coefficients as $3 D$ shape descriptors for protein binding pocket and ligand comparisons. Bioinformatics, 2005. 21(10): p. 2347-2355.

3. Venkatraman, V., et al. "Potential for Protein Surface Shape Analysis Using Spherical Harmonics and 3D Zernike Descriptors." Cell Biochemistry and Biophysics, 2009. 54(1-3): 23-32. 


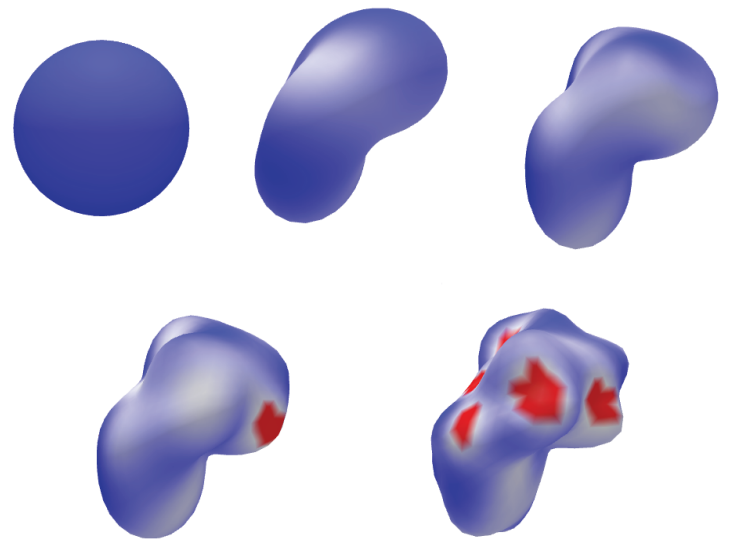

Figure 1. Reconstruction (from the resultant shape coefficients) of a sucrose molecule Hirshfeld surface along with a property on the surface (in this case $\mathrm{d}_{\text {norm }}$ ) for angular momentum $1=1,4,6,8,9$

Keywords: spherical harmonics, Hirshfeld surface, crystal packing, intermolecular interactions, cluster analysis

\section{MS36-P16 Cyclohexylhemicucurbit[8]uril -} a chiral macrocyclic host for anionic guests

Sandra Kaabel ${ }^{1,2}$, Filip Topić ${ }^{2}$, Elena Prigorchenko ${ }^{1}$, Marina Kudrjašova $^{1}$, Elina Kalenius ${ }^{2}$, Lauri Kivijärvi ${ }^{2}$, Riina Aav ${ }^{1}$, Kari Rissanen $^{2}$

1. Department of Chemistry, Tallinn University of Technology, Akadeemia tee 15, 12618 Tallinn, Estonia

2. University of Jyvaskyla, Department of Chemistry, Nanoscience

Center, P.O. Box. 35, FI-40014 University of Jyvaskyla, Finland

email: sandra.kaabel@ttu.ee

Cucurbituril chemistry has gained considerable interest over the last decades. Classical cucurbit $[n]$ uril $(\mathrm{CB}[n])$ homologues, known to encapsulate neutral or positively charged molecules, have already been employed in stimuli responsive systems and in $\mathrm{CB}[n]$ mediated catalysis. ${ }^{[1][2]}$ Accounts of anion binding within $\mathrm{CB}[n]$ homologues are scarce, with the exception of hemicucurbit $[n]$ urils $(\mathrm{HC}[n])$, a sub-group of the $\mathrm{CB}[n]$ family, known to preferentially bind anions. ${ }^{[3][4]}$

Chiral cyclohexylhemicucurbit $[n]$ urils ( $\operatorname{cycHC}[n])$ have been synthesized and investigated by our group. ${ }^{[5][6]}$ The electron-poor cavity of $\operatorname{cycHC}[n]$ renders these macrocycles capable of anion recognition. ${ }^{[7]}$ Our recent efforts to gain insight into the binding of anionic guests by cycHC[8] have resulted in a number of successfully crystallized and structurally characterized complexes. Crystal structures of 1:1 complexes with a number of singly charged tetrahedral and octahedral anions have been successfully obtained, demonstrating the remarkable ability of cycHC[8] to accommodate guests of different shapes and sizes. The flexible nature of the cycHC[8] portals allows for tight sealing of the encapsulated guests. Additionally, the binding of anionic guests to cycHC[8] has been complementarily evaluated by ESI-TOF MS and NMR spectroscopy.

[1] L. Isaacs, Acc. Chem. Res. 2014, 47, 2052-2062. [2] K. I. Assaf, W. M. Nau, Chem. Soc. Rev. 2014, 44, 394-418. [3] Y. Miyahara, K. Goto, M. Oka, T. Inazu, Angew. Chem. Int. Ed. Engl. 2004, 43, 5019-5022. [4] M. A. Yawer, V. Havel, V. Sindelar, Angew. Chem. Int. Ed. Engl. 2015, 54, 276-279. [5] R. Aav, E. Shmatova, I. Reile, M. Borissova, F. Topić, K. Rissanen, Org. Lett. 2013, 15, 3786-3789. [6] M. Fomitšenko, E. Shmatova, M. Öeren, I. Järving, R. Aav, Supramol. Chem. 2014, 26, 698-703. [7] M. Öeren, E. Shmatova, T. Tamm, R. Aav, Phys. Chem. Chem. Phys. 2014, 16, 19198-19205. 